This is a provisional PDF only. Copyedited and fully formatted version will be made available soon.
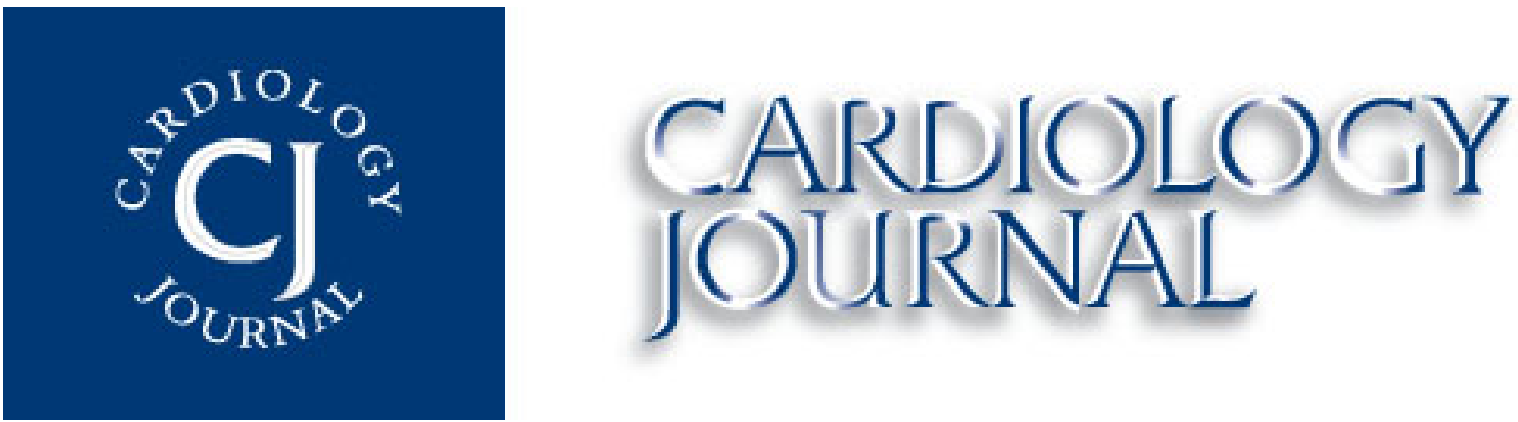

ISSN: 1897-5593

e-ISSN: 1898-018X

\title{
Association of IL-4 gene polymorphisms with Ischemic Heart Failure
}

Author: Nima Rezaei

DOI: $10.5603 /$ J.$a 2013.0049$

Article type: Original articles

Submitted: $2013-02-24$

Accepted: 2013-04-06

Published online: 2013-04-24

This article has been peer reviewed and published immediately upon acceptance.

It is an open access article, which means that it can be downloaded, printed, and distributed freely, provided the work is properly cited.

Articles in "Cardiology Journal" are listed in PubMed. 


\section{Association of IL-4 gene polymorphisms with Ischemic Heart Failure}

Mohammad Jafar Mahmoudi ${ }^{1}$, Mona Hedayat ${ }^{2}$, Mohammad Taghvaei $^{3}$, Ebrahim Nematipour ${ }^{4}$, Elham Farhadi ${ }^{5}$, Nilufar Esfahanian ${ }^{3}$, Maryam Sadr ${ }^{3}$, Maryam Mahmoudi ${ }^{6}$, Keramat Nourijelyani $^{7}$, Ali Akbar Amirzargar ${ }^{3,8}$, Nima Rezaei ${ }^{3,8,9}$

1. Division of Cardiology, Department of Internal Medicine, School of Medicine, Tehran University of Medical Sciences, Tehran, Iran

2. Division of Immunology, Boston Children's Hospital, Harvard Medical School, Boston, MA, USA

3. Molecular Immunology Research Center, Tehran University of Medical Sciences, Tehran, Iran

4. Tehran Heart Center, Tehran University of Medical Sciences, Tehran, Iran

5. Hematology Department, School of Allied Medical Science, Tehran University of Medical Sciences, Tehran, Iran

6. School of Nutrition and Dietetics, Tehran University of Medical Sciences, Tehran, Iran 7. Department of Epidemiology and Biostatistics, School of Public Health, Tehran University of Medical Sciences, Tehran, Iran

8. Department of Immunology, School of Medicine, Tehran University of Medical Sciences, Tehran, Iran

9. Research Center for Immunodeficiencies, Children's Medical Center, Tehran University of Medical Sciences, Tehran, Iran

Correspondence: Nima Rezaei, $\mathrm{MD}, \mathrm{PhD}$

Address: Children’s Medical Center Hospital, Dr Gharib St, Keshavarz Blvd, Tehran, Iran

E-mail: rezaei nima@tums.ac.ir

Running Title: IL-4 SNPs in Ischemic Heart Failure 


\begin{abstract}
Background: As of the potential immunomodulatory effects of interleukin-4 (IL-4) and its importance in inhibiting the production of proinflammatory cytokines by monocytes and activated T cells, the IL-4 gene polymorphisms were investigated in a group of patients with chronic heart failure (CHF) due to ischemic heart disease.
\end{abstract}

Methods: Forty three patients with ischemic heart failure were enrolled in this study and compared with 139 healthy individuals. The allele and genotype frequency of 3 single nucleotide polymorphisms (SNPs) within the IL-4 gene were determined.

Results: The frequency of the IL-4 -590/T allele in the patient group was significantly higher than in the control group $(p<0.0001)$. The most frequent genotypes in patients with ischemic heart failure were IL-4 (-590) CC $(p<0.0001)$, IL-4 (-33) CC $(p=0.021)$, and IL-4 (-33) TT $(p<0.0001)$. The frequency of the following genotypes was significantly lower in patients compared to controls: IL-4 (-1098) TG ( $p=0.035)$, IL-4 (-590) TC $(p<0.0001)$, and IL-4 (-33) TC $(p<0.0001)$. The most frequent IL-4 haplotypes in the patient group, which were significantly higher than in the control group, were TCC $(p<0.0001)$, TCT ( $p=0.0242$ ), and GCT ( $p=0.0108)$ haplotypes. In contrast, the frequencies of the following haplotypes in the patient group were significantly lower than the controls: GCC $(p=0.032)$, TTT $(p=0.0268)$, and TTC $(p=0.0399)$.

Conclusions: Certain alleles, genotypes, and haplotypes in IL-4 gene were overrepresented in patients with ischemic heart failure, which may, in turn, predispose individuals to this disease.

Key words: Heart failure, gene polymorphisms, interleukin-4 


\section{Introduction}

Chronic heart failure (CHF) is a clinical syndrome characterized by progressive ventricular dilation, depressed contractile function, and increased morbidity and mortality . Despite our increasing knowledge of the molecular, genetic, and biochemical pathways involved in the pathogenesis of CHF, much is left to be understood.

Increased circulating and intracardiac levels of proinflammatory cytokines have been reported repeatedly in patients with CHF, and more important, their contributions in mediating cardiac adaptive and maladaptive responses have been a topic of intensive research .

Given the potential immunomodulatory effects of IL-4 and its importance in inhibiting the production of proinflammatory cytokines by monocytes and activated T cells , IL-4 gene polymorphisms altering cytokine production might affect individual susceptibility to ischemic heart failure. The association of IL4 gene polymorphisms and a number of diseases with possible underlying immune disturbances have already been investigated . In the present study, three cytokine single nucleotide polymorphisms situated at positions $-1098(\mathrm{G} / \mathrm{T}),-590(\mathrm{C} / \mathrm{T})$, and $-33(\mathrm{C} / \mathrm{T})$ in the promoter region of the $\mathrm{IL}-4$ gene were investigated in Iranian patients with ischemic heart failure. 


\section{Materials and Methods}

\section{Subjects}

In the present study, a total of 43 Iranian patients with end-stage ischemic heart failure (mean age $60.05 \pm$ 11.97; 34 men, 9 women) with angiographically significant coronary artery disease, defined as $\geq 50 \%$ diameter stenosis in at least one of the major coronary arteries, were enrolled. The diagnosis of end-stage heart failure was based on impaired left ventricular systolic function (left ventricular ejection fraction $\leq$ $40 \%$ ) and left ventricular dilation (left ventricular end-diastolic diameter $>5.5 \mathrm{~cm}$ ) on echocardiography. All patients underwent transthoracic echocardiography and cardiac catheterization. Subjects with chronic lung disease, malignancies, recent myocardial infarction, and acute decompensated heart failure within 3 months before recruitment were excluded. Eligible patients were in stable clinical condition and received conventional medical therapy for at least 3 months.

One hundred and thirty nine control subjects (mean age $45.63 \pm 10.84 ; 101$ men, 39 women) were randomly selected from healthy volunteers, as previously described . Written informed consent was obtained from all participants prior to blood sampling. This study was approved by the Ethical Committee of Tehran University of Medical Sciences.

\section{Genotyping}

Cytokine typing was performed on genomic DNA by polymerase chain reaction with sequence-specific primers (PCR-SSP) assay (PCR-SSP kit, Heidelberg University, Heidelberg, Germany), as previously described in detail . Briefly, amplification was carried out using a thermal cycler Techne Flexigene apparatus (Rosche, Cambridge, UK). The presence or absence of PCR products was visualized by 2\% agarose gel electrophoresis. All individuals were genotyped for 3 polymorphic sites in IL-4 gene: -1098 $\mathrm{T} / \mathrm{G},-590 \mathrm{C} / \mathrm{T}$, and $-33 \mathrm{C} / \mathrm{T}$.

\section{Statistical Analysis}


Statistical analyses were performed with GraphPad Prism 5.00 for Windows (Graphpad Software). Allele, genotype, and haplotype frequencies for all cytokine gene polymorphisms were calculated by direct counting. Frequencies of alleles, genotypes, and haplotypes were compared between the patient and control groups using the Fisher's exact test. The odds ratio and 95\% confidence intervals were calculated. 


\section{Results}

\section{Alleles and Genotype Frequencies}

IL-4 allelic and genotype frequencies in patients with ischemic heart failure and healthy controls are presented in Table 1.

The frequency of the IL-4 -590/T allele in the patient group was significantly higher than in the control group (73.3\% in patients vs. $46.4 \%$ in controls, $p<0.0001)$. The most frequent genotypes in patients with ischemic heart failure were IL-4 CC genotype at position -590 (51.1\% in patients vs. $7.2 \%$ in controls, $p<$ 0.0001), IL-4 CC genotype at position -33 (65\% in patients vs. $43.9 \%$ in controls, $p=0.021$ ), and IL-4 TT genotype at position -33 (15\% in patients vs. $0 \%$ in controls, $p<0.0001)$. In contrast the frequency of the following genotypes in the patient group was significantly lower than in the control group: IL-4 TG at position -1098 (39.6\% in patients vs. 59\% in controls, $p=0.035)$, IL-4 TC at position -590 (44.2\% in patients vs. $92.8 \%$ in controls, $p<0.0001$ ), and IL-4 TC at position -33 (20\% in patients vs. $56.1 \%$ in controls, $p<0.0001)$.

\section{Haplotype Frequencies}

The frequency of haplotypes of IL-4 (-1098, -590, -33) in patients with ischemic heart failure and healthy controls are shown in Table 1. The most frequent haplotype in our patients was IL-4 TCC which was significantly higher than in the control group (47.5\% in patients vs. $23.4 \%$ in controls, $p<0.0001)$. In addition IL-4 TCT (5\% in patients vs. $0.7 \%$ in controls, $p=0.0242)$ and GCT (3.75\% in patients vs. $0 \%$ in controls, $p=0.0108$ ) haplotypes were significantly more common in the patient group compared to controls. In contrast, the frequencies of the following haplotypes in the patient group were significantly lower than the controls: IL-4 GCC (17.5\% in patients vs. $30 \%$ in controls, $p=0.032)$, TTT $(15 \%$ in patients vs. $27.3 \%$ in controls, $p=0.0268)$, and TTC (8.75\% in patients vs. $18.3 \%$ in controls, $p=0.0399)$. 


\section{Discussion}

In the present study, the frequency of the IL-4/C allele at position -590 was decreased significantly in patients with ischemic heart failure; however, the frequency of the CC genotype at the same position was significantly overrepresented in patients compared to controls. In addition, while less than half of patients had the TC genotype at position -590, it was found in the majority of the healthy controls. It has been shown that the $\mathrm{C}$ to $\mathrm{T}$ exchange at position -590 of the IL-4 gene is associated with increased IL-4 production, with the CC, TC, and TT genotypes being associated with low, intermediate, and high IL-4 production, respectively . With regard to IL-4 -590 C/T polymorphism, Bijlsma FJ et al. reported no correlation between IL-4 promoter gene polymorphism at position -590 and heart failure, and allograft rejection after heart transplantation. However, in patients with the CC genotype (low IL-4 production), receiving a heart from a T-positive donor reduced the incidence of rejection substantially. It has been concluded that high IL-4 production within -590/T-positive donor hearts protects against rejection .

For IL-4 at position -33, there was no significant difference in allele frequencies between our patients and controls. However, we found significant positive associations with the CC and TT genotypes in our patients compared to controls; while the TC genotype at the same position was decreased significantly in patients than controls. It has been shown that IL-4 promoters harboring $-33 / C$ allele plays a significant role in the activation of IL-4 transcription, and that the homozygous variant of IL-4 (-33) CC is associated with increased IL-4 production . In this study, the CC genotype at position -33 was the most frequent genotype in both groups of patients and controls; however, while none of the healthy controls were found to have the TT genotype, it was significantly overrepresented in patients with ischemic heart failure.

The IL-4 (-1098, 0590, -33) haplotypes GCC, TTT, TCC, and TTC are the most common haplotypes in normal Iranian population . In the present study, the TCC haplotype was the most frequent haplotype in patients with ischemic heart failure and was significantly overrepresented in patients compared to controls. However, the GCC, TTT, and TTC haplotypes in the patient group were significantly less 
frequent than in healthy controls. On the other hand, the IL-4 TCT and GCT haplotypes were significantly overrepresented in patients than controls. Given the fact that IL-4 (-1098) T/G polymorphism was found to have no correlation with serum IL-4 level , and that the IL-4 -590/C and -33/T alleles are associated with decreased IL-4 production , it could be suggested that there is a tendency towards lowered IL-4 production in at least a subset of patients with ischemic heart failure. However, given the small number of patients in our study, we must view these results with caution.

IL-4 is an important mediator of T-helper 2 (Th2) immune responses by inducing differentiation of naive $\mathrm{CD}^{+} \mathrm{T}$ cells to Th2 cells , regulating humoral immune responses , and inhibiting the production of Th1 cytokines by monocytes and activated T cells . Moreover, IL-4 stimulates fibroblast proliferation and collagen synthesis, and thus has been implicated in the progression of fibrosis . Increased urinary excretion of circulating procollagen type-III amino-terminal propeptide (PIIINP) has been known as a marker of ongoing fibrotic processes . Likewise, increased serum PIIINP levels have also been demonstrated in patients with ischemic and dilated cardiomyopathy . It has been demonstrated that IL-4, most likely produced by mast cells in the heart during pressure overload, significantly contribute to cardiac fibrosis . In patients with heart failure, the urinary IL-4 level correlated with left ventricular endsystolic volume index, left ventricular end-diastolic volume index, and with PIIINP . The correlation between urinary IL-4 level and cardiac fibrosis and remodeling was found to be stronger in patients with hypertensive cardiomyopathy . At the present time, the pathologic significance of IL-4 in the development and progression of chronic heart failure is not well understood. The majority of studies failed to demonstrate any significant difference in IL-4 producing peripheral CD4 ${ }^{+} \mathrm{T}$ cells and IL-4 levels between the two groups of patients and controls .

To the best of our knowledge, we demonstrated for the first time the association between certain allele, genotype, and haplotype frequencies in IL-4 gene with ischemic heart failure. Further studies will provide additional understanding of the possible role of cytokine gene polymorphisms in the development and progression of ischemic heart failure. 


\section{Acknowledgement}

This study was supported by grant from Tehran University of Medical Sciences and Health Services (87-04-93-9584). 


\section{References}

1. Lloyd-Jones, D.M., et al., Lifetime risk for developing congestive heart failure: the Framingham Heart Study. Circulation, 2002. 106(24): p. 3068-72.

2. Levine, B., et al., Elevated circulating levels of tumor necrosis factor in severe chronic heart failure. N Engl J Med, 1990. 323(4): p. 236-41.

3. Aukrust, P., et al., Cytokine network in congestive heart failure secondary to ischemic or idiopathic dilated cardiomyopathy. Am J Cardiol, 1999. 83(3): p. 376-82.

4. Hirota, H., et al., Circulating interleukin-6 family cytokines and their receptors in patients with congestive heart failure. Heart Vessels, 2004. 19(5): p. 237-41.

5. Satoh, S., et al., Increased productivity of tumor necrosis factor-alpha in helper $T$ cells in patients with systolic heart failure. Int J Cardiol, 2006. 111(3): p. 405-12.

6. El-Menyar, A.A., Cytokines and myocardial dysfunction: state of the art. J Card Fail, 2008. 14(1): p. 61-74.

7. Hedayat, M., et al., Proinflammatory cytokines in heart failure: double-edged swords. Heart Fail Rev, 2010. 15(6): p. 543-62.

8. Essner, R., et al., IL-4 down-regulates IL-1 and TNF gene expression in human monocytes. J Immunol, 1989. 142(11): p. 3857-61.

9. Damle, N.K. and L.V. Doyle, Distinct regulatory effects of IL-4 and TNF-alpha during CD3-dependent and CD3-independent initiation of human T-cell activation. Lymphokine Res, 1989. 8(2): p. 85-97.

10. Rezaei, N., et al., Association of IL-4 and IL-10 gene promoter polymorphisms with common variable immunodeficiency. Immunobiology, 2010. 215(1): p. 81-7.

11. Shahram, F., et al., Association of interleukin-2, interleukin-4 and transforming growth factor-beta gene polymorphisms with Behcet's disease. Clin Exp Rheumatol, 2011. 29(4 Suppl 67): p. S28-31.

12. Amirzargar, A.A., et al., Cytokine gene polymorphism in Iranian patients with chronic myelogenous leukaemia. Int J Immunogenet, 2005. 32(3): p. 167-71.

13. Amirzargar, A.A., et al., Cytokine single nucleotide polymorphisms in Iranian patients with pulmonary tuberculosis. Eur Cytokine Netw, 2006. 17(2): p. 84-9.

14. Amirzargar, A.A., et al., Polymorphisms in IL4 and iLARA confer susceptibility to asthma. J Investig Allergol Clin Immunol, 2009. 19(6): p. 433-8.

15. Amirzargar, A.A., et al., Cytokine single nucleotide polymorphisms in Iranian populations. Eur Cytokine Netw, 2008. 19(2): p. 104-12.

16. Rosenwasser, L.J., et al., Promoter polymorphisms in the chromosome 5 gene cluster in asthma and atopy. Clin Exp Allergy, 1995. 25 Suppl 2: p. 74-8; discussion 95-6.

17. Bijlsma, F.J., et al., Donor interleukin-4 promoter gene polymorphism influences allograft rejection after heart transplantation. J Heart Lung Transplant, 2002. 21(3): p. 340-6.

18. Kim, B.S., et al., Effect of single nucleotide polymorphisms within the interleukin-4 promoter on aspirin intolerance in asthmatics and interleukin-4 promoter activity. Pharmacogenet Genomics, 2010. 20(12): p. 748-58.

19. Gervaziev, Y.V., V.A. Kaznacheev, and V.B. Gervazieva, Allelic polymorphisms in the interleukin-4 promoter regions and their association with bronchial asthma among the Russian population. Int Arch Allergy Immunol, 2006. 141(3): p. 257-64. 
20. Abehsira-Amar, O., et al., IL-4 plays a dominant role in the differential development of Tho into Th1 and Th2 cells. J Immunol, 1992. 148(12): p. 3820-9.

21. Rogge, L., A genomic view of helper T cell subsets. Ann N Y Acad Sci, 2002. 975: p. 5767.

22. Timonen, P., et al., Cytokines, interstitial collagen and ventricular remodelling in dilated cardiomyopathy. Int J Cardiol, 2008. 124(3): p. 293-300.

23. Soylemezoglu, O., et al., Urinary and serum type III collagen: markers of renal fibrosis. Nephrol Dial Transplant, 1997. 12(9): p. 1883-9.

24. Teppo, A.M., et al., Urinary amino-terminal propeptide of type III procollagen (PIIINP) as a marker of interstitial fibrosis in renal transplant recipients. Transplantation, 2003. 75(12 ): p. 2113-9.

25. Rivera, M., et al., [Myocardial remodeling and immunologic activation in patients with heart failure]. Rev Esp Cardiol, 2006. 59(9): p. 911-8.

26. Kanellakis, P., et al., A pro-fibrotic role for interleukin-4 in cardiac pressure overload. Cardiovasc Res, 2012. 95(1): p. 77-85.

27. Rosello-Lleti, E., et al., [Interleukin-4 and cardiac fibrosis in patients with heart failure]. Rev Esp Cardiol, 2007. 60(7): p. 777-80.

28. Fukunaga, T., et al., Expression of interferon-gamma and interleukin-4 production in CD4+ T cells in patients with chronic heart failure. Heart Vessels, 2007. 22(3): p. 178-83.

29. Cheng, X., et al., [The significance of Th1/Th2 function imbalance in patients with postinfarction cardiac insufficiency]. Zhonghua Xin Xue Guan Bing Za Zhi, 2005. 33(6): p. 526-8.

30. Fukunaga, T., et al., Relation between CD4+ T-cell activation and severity of chronic heart failure secondary to ischemic or idiopathic dilated cardiomyopathy. Am J Cardiol, 2007. 100(3): p. 483-8. 
Table 1. Comparisons of allele, genotype and haplotype frequencies between patients with ischemic heart failure and controls.

\begin{tabular}{|c|c|c|c|c|c|c|}
\hline Cytokine & Position & $\begin{array}{l}\text { Alleles/ } \\
\text { Genotypes/ } \\
\text { Haplotypes }\end{array}$ & $\begin{array}{l}\text { Patients }(n=43 \\
\text { ) } \\
\text { N (\%) }\end{array}$ & $\begin{array}{l}\text { Controls (n=139 } \\
\text { ) } \\
\text { ( (\%) }\end{array}$ & P-value & Odds Ratio (95\% CI) \\
\hline \multirow{15}{*}{ IL-4 } & \multirow{5}{*}{-1098} & G & $19(22.1)$ & $84(30.2)$ & 0.171 & 0.65 (0.37 to 1.16$)$ \\
\hline & & $\mathrm{T}$ & 67 (77.9) & $194(69.8)$ & 0.171 & 1.53 (0.86 to 2.70$)$ \\
\hline & & GG & $1(2.3)$ & $1(0.7)$ & 0.418 & $3.29(0.20$ to 53.71$)$ \\
\hline & & TG & 17 (39.6) & $82(59)$ & $0.035 *$ & $0.45(0.23$ to 0.91$)$ \\
\hline & & TT & $25(58.1)$ & $56(40.3)$ & 0.053 & 2.06 (1.03 to 4.12$)$ \\
\hline & \multirow[t]{5}{*}{-590} & $\mathrm{C}$ & $23(26.7)$ & 149 (53.6) & $<0.0001^{*}$ & 0.32 (0.19 to 0.54$)$ \\
\hline & & $\mathrm{T}$ & $63(73.3)$ & $129(46.4)$ & $<0.0001 *$ & 3.16 (1.86 to 5.39$)$ \\
\hline & & CC & $22(51.1)$ & $10(7.2)$ & $<0.0001 *$ & 13.51 (5.61 to 32.53$)$ \\
\hline & & TC & $19(44.2)$ & $129(92.8)$ & $<0.0001 *$ & 0.06 (0.03 to 0.15$)$ \\
\hline & & TT & $2(4.7)$ & $0(0)$ & 0.055 & 16.81 (0.79 to 357.3$)$ \\
\hline & \multirow[t]{5}{*}{-33} & $\mathrm{C}$ & $60(75)$ & 200 (71.9) & 0.670 & 1.17 (0.66 to 2.07 ) \\
\hline & & $\mathrm{T}$ & $20(25)$ & $78(28.1)$ & 0.670 & $0.86(0.48$ to 1.51$)$ \\
\hline & & CC & $26(65)$ & 61 (43.9) & $0.021 *$ & 2.38 (1.14 to 4.93$)$ \\
\hline & & TC & $8(20)$ & 78 (56.1) & $<0.0001 *$ & $0.20(0.08$ to 0.45$)$ \\
\hline & & TT & $6(15)$ & $0(0)$ & $<0.0001 *$ & 52.57 (2.89 to 956.5$)$ \\
\hline \multirow[t]{8}{*}{ IL-4 } & \multirow{8}{*}{$\begin{array}{l}-1098, \\
-590,-33\end{array}$} & GCC & $14(17.5)$ & $83(30)$ & $0.032 *$ & 0.5 (0.27 to 0.94$)$ \\
\hline & & TTT & $12(15)$ & $76(27.3)$ & $0.027 *$ & $0.47(0.24$ to 0.91$)$ \\
\hline & & TCC & $38(47.5)$ & 65 (23.4) & $<0.0001 *$ & 2.97 (1.76 to 4.98$)$ \\
\hline & & TTC & $7(8.75)$ & $51(18.3)$ & 0.039* & 0.43 (0.19 to 0.98$)$ \\
\hline & & TCT & $4(5)$ & $2(0.7)$ & $0.024 *$ & 7.26 (1.31 to 40.43$)$ \\
\hline & & GTT & $1(1.25)$ & $1(0.3)$ & 0.397 & 3.51 (0.22 to 56.73$)$ \\
\hline & & GCT & $3(3.75)$ & $0(0)$ & $0.011 *$ & 25.15 (1.29 to 492.6$)$ \\
\hline & & GTC & $1(1.25)$ & $0(0)$ & 0.223 & 10.51 ( 0.42 to 260.7$)$ \\
\hline
\end{tabular}

